\title{
VITAMIN D3 SUPPLEMENTATION IN PATIENTS WITH SEVERE SLE DISEASE: BENEFITIAL EFFECTS WITH HIGH OR ROUTINE DOSES IN A RANDOMIZED STUDY
}

Lisbeth Arancibia Aguila ${ }^{1}$, Felipe Mendonça de Santana ${ }^{1}$, Michelle Remião Ugolini-Lopes ${ }^{1}$, Luciana Parente Costa Seguro' ${ }^{1}$ Rosa Maria Rodrigues Pereira ${ }^{1, *}$

1.Universidade de São Paulo, São Paulo (SP), Brazil.

*Corresponding author: rosamariarp@yahoo.com

\section{BACKGROUND}

Systemic lupus erythematosus (SLE) is a systemic autoimmune disease with pleotropic and potentially serious manifestations. Studies indicate a potential role of vitamin D in SLE disease activity but studies on inpatients with SLE have not been performed. The aim of our study was to evaluate the effect of high dose vitamin D3 oral supplementation versus placebo, in patients with severe SLE disease activity submitted to corticosteroid pulse therapy.

\section{MATERIALS AND METHODS}

Forty SLE patients ( $85 \%$ women), admitted to a tertiary rheumatology ward, were included in a randomized, double-blind placebo-controlled trial. One group was submitted to placebo ( $G 1, n=20)$ and the other group to vitamin D3 100,000 IU/ week (G2, $n=20$ ). Both groups received routine dose of vitamin D3 (7,000 IU/week), as well as standard treatment for SLE activity. All patients (baseline SLEDAI - G1: $17.6 \pm 10.06$ vs. G2: $19.3 \pm 8.07, p=0.57$ ) received methylprednisolone pulse therapy (0.5-1 g/day, 3-5 days). Demographic and laboratory variables were collected at baseline and at months 1,3 and 6 of follow-up. Fatigue, quality of life and functionality were assessed by appropriate questionnaires at baseline and follow-up. The outcomes of interest in the study were changes in clinical variables and laboratory tests related to disease activity, assessed in the form of delta ( $\Delta=$ final value-baseline value). The total follow-up was 6 months. Student's T test, Welch's T test, Mann-Whitney, Spearman correlation and ROC curves were used, as appropriate. P-value $<0.05$ was considered statistically significant.

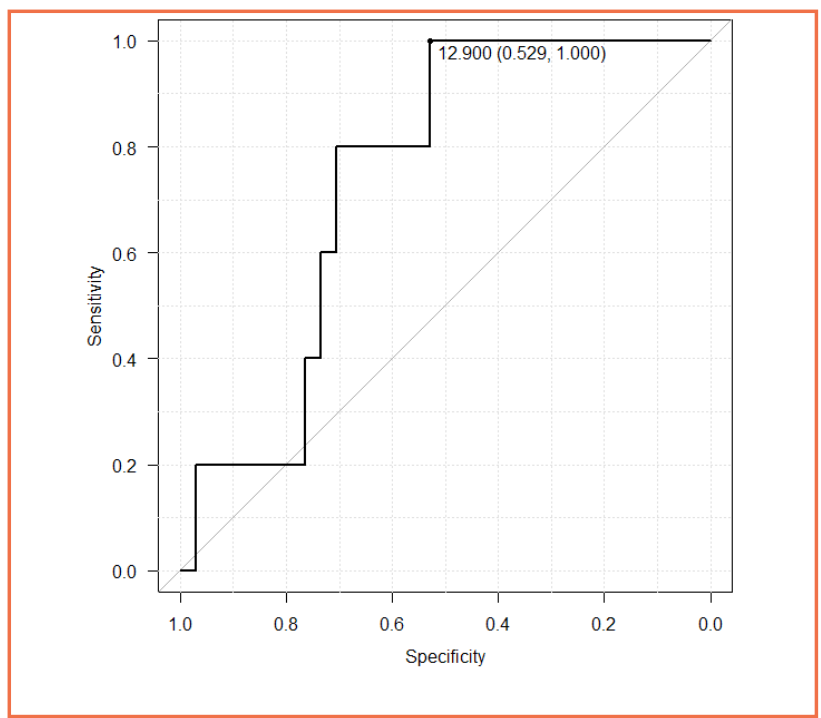

Figure 1. ROC curve for performance of $\triangle 25 \mathrm{OHVitD}$ to predict $\mathrm{C} 3$ normalization in the entire cohort.

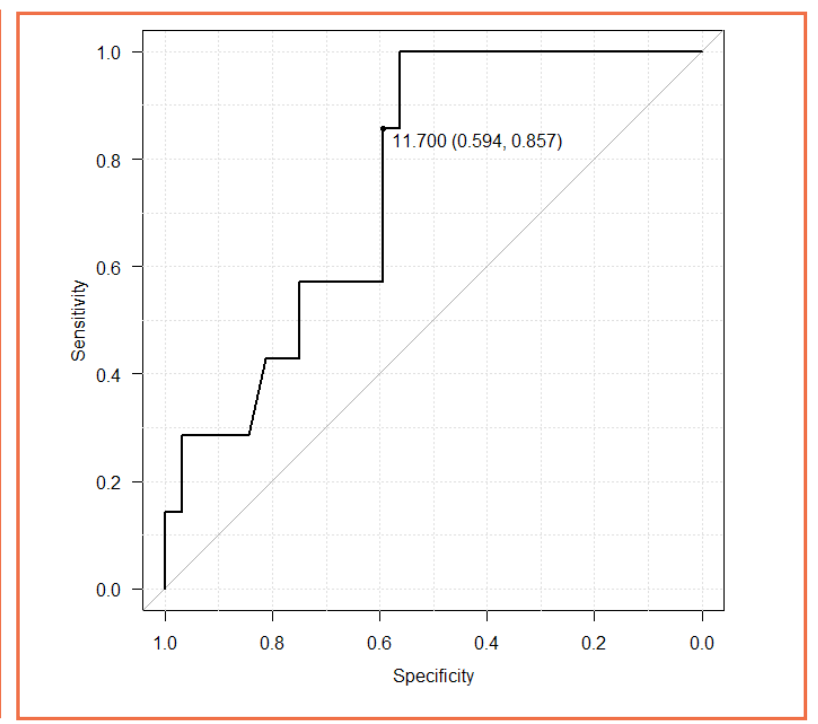

Figure 2. ROC curve for performance of $\triangle 25 O H V i t D$ to predict $C 4$ normalization in the entire cohort. 


\section{RESULTS}

Both groups were homogeneous for most of the baseline variables. After supplementation, statistical significance was found in serum $\Delta$ calcium (G1: $0.08 \pm 0.23$ vs. G2: $0.27 \pm 0.30 \mathrm{mg} / \mathrm{dL}, p=0.037$ ), serum $\triangle 25 \mathrm{OHD}$ (G1: 7.95 [IQ 14.95-3.0] vs. G2: 15.4 [IQ 31.0-6.5] ng/mL, $p=0.028$ ) and a positive trend in $\Delta C 4(G 1: 3.27 \pm 7.32$ vs. G2: $7.38 \pm 7.59 \mathrm{mg} / \mathrm{dL}, p=0.093)$. There were no significant differences between randomized groups for other variables. For a second analysis, assessing the entire sample in spite of group allocation, $\triangle 25 \mathrm{OHD}$ showed significant positive correlation with $\triangle$ disease activity parameters, namely $\triangle \mathrm{C} 3$ ( $r$ ho $=0.48$, $p=0.001), \Delta C 4$ ( $r h o=0.35, p=0.025)$, $\Delta$ anti-DNAds ( $r h o=-0.35, p=0.026$ ). The analysis by ROC curve of the performance of $\triangle 25 \mathrm{OHD}$ to discriminate decreased levels of C3 (Fig. 1) and C4 (Fig. 2) showed an area under the curve with moderate performance (C3- AUC 0.741, cut-off of $\triangle 12.9$ ) (C4- AUC 0.757, cut-off of $\triangle 11.7$ ).

\section{CONCLUSION}

Maintenance of adequate serum 250HD levels, whether by supplementation of high or routine doses, was associated with a positive effect on laboratory parameters in severe SLE patients receiving high dose parenteral corticosteroid. 